\title{
Gabapentinoids for Pruritus in Older Adults: A Narrative Review
}

\author{
Shreya A. Sreekantaswamy · Nicholas Mollanazar · Daniel C. Butler
}

Received: January 15, 2021 / Published online: March 15, 2021

(C) The Author(s) 2021

\section{ABSTRACT}

There is currently no standardized algorithm for the treatment of chronic pruritus (CP), or itch lasting more than 6 weeks, in adults aged $\geq 65$ years. The antiepileptic agents gabapentin and pregabalin, however, are gaining popularity in the dermatologic community for their efficacy in treating $\mathrm{CP}$ of neuropathic origin. Yet the lack of literature specifically looking at the safety and efficacy of these medications in older adults results in limited guidance for providers in the safe use of gabapentinoids. In this paper we discuss special considerations and recommendations for treating older adults with gabapentin and pregabalin and explore the possibility for these drugs to ameliorate $\mathrm{CP}$ of multiple etiologies.

Keywords: Chronic pruritus; Gabapentin; Gabapentinoid; Itch; Older adult; Pregabalin

S. A. Sreekantaswamy · D. C. Butler $(\bowtie)$

San Francisco Department of Dermatology,

University of California, San Francisco, CA, USA

e-mail: Daniel.Butler@UCSF.edu

S. A. Sreekantaswamy

School of Medicine, University of Utah, Salt Lake

City, UT, USA

N. Mollanazar

Department of Dermatology, University of

Pennsylvania, Philadelphia, PA, USA

\section{Key Summary Points}

Gabapentin and pregabalin are safe and efficacious options to treat older adults with chronic pruritus.

Prior to gabapentinoid initiation, the patient should be screened for: history of kidney disease; COPD or other lung disorders; current opioid medication use; history of falls, lightheadedness, or vertigo; history of mood disorders, including depression and suicidal ideation; history of recreational drug usage.

Common side effects of both gabapentin and pregabalin that should be screened for after initiation or dose change include: changes in mood or increased depression; anxiety, or suicidal ideation; dizziness, fatigue, or blurred vision; difficulty with balance or coordination; edema in extremities.

The goal of treatment is to control itch at night: start with $100 \mathrm{mg}$ at 8:00 p.m. and then increase dose to $300 \mathrm{mg}$ before adding a second evening dose at 5:00 p.m. 


\section{DIGITAL FEATURES}

This article is published with digital features, including a summary slide, to facilitate understanding of the article. To view digital features for this article go to https://doi.org/10.6084/ m9.figshare.14152370.

\section{INTRODUCTION}

The treatment of chronic pruritus (CP), or itch lasting longer than 6 weeks, has long challenged the dermatologist, especially in older adults, defined as age $\geq 65$ years. One class of drugs gaining popularity among dermatologists for its efficacy in treating and managing CP are the gabapentinoids. This drug class includes gabapentin and pregabalin, and although they were initially developed as antiepileptics, data have revealed that they show promise for the treatment of CP.

The reported prevalence of $\mathrm{CP}$ among older adults varied from 11.5 to $38.9 \%$ of the respective study populations [1]. The International Forum for the Study of Itch (IFSI) classifies CP into six categories of pruritogenic etiology: (1) dermatologic diseases, (2) systemic diseases, including diseases of pregnancy and drug-induced pruritus, (3) neurological, (4) psychiatric/ psychosomatic diseases, (5) mixed etiology, and (6) unknown/other [2]. Although the origin of pruritus can be unknown in up to $20 \%$ of cases [3], the running theory is that CP in the older adult is often multifactorial [4] and that this mixed etiology is likely further potentiated by comorbidity and polypharmacy in this population [5]. Mixed-etiology CP is thought to result from a combination of the following three processes: disruption of barrier function (e.g., xerosis, ichthyosis), immunologic dysregulation (e.g., bullous pemphigoid), or neurologic changes (e.g., notalgia paresthetica) [4]. Neuropathic itch is thought to be a result of neuronal sensitization from disorders of either the peripheral or central nervous systems. Pruritusinducing conditions of the peripheral nervous system may be divided into those caused by nerve fiber compression-with pruritus localized to a dermatome-versus those caused by nerve fiber degeneration, with pruritus either localized or generalized. Classic conditions that can cause neuropathic pruritus include brachioradial pruritus, notalgia paresthetica, and postherpetic neuralgia [6].

The initial treatment of $\mathrm{CP}$ varies across healthcare providers. Many dermatologists consider topical steroids and moisturizers as first-line agents and, despite evidence to the contrary, antihistamines as second-line options [7]. This therapeutic pattern is not supported by robust literature. However, antihistamine use for CP in older adults is ubiquitous despite the Beers criteria recommending against their use [8]. The Beers criteria are a list published by the American Geriatrics Society which specifies medications that can potentially be used inappropriately in older adults. The reasons cited against antihistamine use in this population include anticholinergic effects and toxicity, such as confusion, dry mouth, and constipation [8]. In addition, investigations on the neural pathways of $\mathrm{CP}$ have shown that $\mathrm{CP}$ does not involve the histaminergic pathways that drive acute pruritus [9].

A 2019 study conducted in the Netherlands found that only $38.3 \%$ of 193 healthcare providers surveyed prescribed gabapentinoids for $\mathrm{CP}$, with $86.5 \%$ of those who did not saying that their main reason was due to lack of knowledge or experience with the medications. Two respondents also stated that they were unaware that gabapentin or pregabalin could even be used to treat CP [10]. Due to these inconsistencies, potential dangers, and lack of awareness, dermatologists need to investigate and standardize CP treatments that are both effective and safe in older adults. In this review we discuss the safety and efficacy of the gabapentinoids, and advocate for their use not just in neuropathic, but also in multifactorial, itch.

\section{METHODS}

The primary aim of this review was to evaluate the safety and efficacy of gabapentinoid use for $\mathrm{CP}$ in older adults, and to provide recommendations for gabapentin and pregabalin use in 
this patient population. On 10 November 2020, we conducted a search of the PubMed/MEDLINE databases using the search terms "gabapentin chronic pruritus," "gabapentin itch," "pregabalin chronic pruritus," "pregabalin itch," "gabapentinoid chronic pruritus," "gabapentinoid itch," "gabapentin older adult," and "pregabalin older adult." We included retrospective and prospective studies as well as general and systematic reviews that reported validated clinical information on gabapentinoid use in older adults. We also evaluated the US Food and Drug Administration (FDA) labels of all brand name gabapentin and pregabalin medications to review data on their safety and efficacy and recommendations for older adults.

\section{Compliance with Ethics Guidelines}

This article is based on previously conducted studies and does not contain any studies with human participants or animals performed by any of the authors.

\section{GABAPENTIN AND PREGABALIN MECHANISM OF ACTION AND USE IN ITCH}

Gabapentin (brand names Neurontin [Pfizer Inc., New York, NY, USA], Gralise [Almatica Pharma, Pine Brook, NJ, USA], and Horizant [Patheon Inc., Thermo Fisher Scientific, Waltham, MA, USA]) and pregabalin (brand name Lyrica [Pfizer Inc., New York, NY, USA]) were both initially developed as antiepileptics but have since been approved to treat neuropathic pain. Both drugs have several common off-label uses, and in the world of dermatology, they are used to treat CP [11].

Gabapentinoids are analogs of the inhibitory neurotransmitter gamma-aminobutyric acid (GABA), but do not affect GABA binding, uptake, or degradation. Although the mechanism of action for these drugs is yet to be fully elucidated, both bind with high affinity to the $\alpha 2 \delta$ subunit of voltage-gated calcium channels in the nervous system. This binding is thought to increase the threshold for neuronal excitation, thereby decreasing the release of excitatory neurotransmitters [12]. Research has shown that the $\alpha 2 \delta$ subunit in particular is upregulated at the site of peripheral nerve injury, which might help explain its selectivity in treating neuropathic pain [13].

With regards to $C P$, the efficacy of gabapentin and pregabalin in modulating itch may be due to the fact that the voltage-gated calcium channels they affect regulate the release of substance $\mathrm{P}$ (SP) and calcitonin gene-related peptide (CGRP) [14]. SP and CGRP are neuropeptides that cause neurogenic inflammation via local vasodilation and mast cell degranulation, and although SP and CGRP are classically released by the histaminergic nerves that are implicated in acute itch, SP and one of its receptors, neurokinin 1 receptor (NK1R), have attracted the attention of the scientific community due to their involvement in CP [9]. Pregabalin and gabapentin have been shown to reduce the release of SP and CGRP from inflamed spinal tissue in in vitro and in vivo rat models $[15,16]$, supporting the hypothesis that the gabapentinoids' role in itch could be due to their modulation of these two key inflammatory neuropeptides.

One of the earliest reports on the use of gabapentin for neuropathic itch was that of Bueller et al. [17] in 1999, who treated a 54-yearold woman with brachioradial pruritus. At the time of their report she had remained asymptomatic for 4 months on a schedule of gabapentin $300 \mathrm{mg}$ six times daily [17]. Since then, gabapentin, and later pregabalin, have been used in the management of CP $[18,19]$. Although still an off-label use, treatment of neuropathic pruritus with gabapentinoids is gaining traction among the dermatologic community [3]. Interestingly, one of the major pruritic conditions treated by gabapentin and pregabalin is uremic pruritus in patients with chronic kidney disease (CKD) [20]. Although uremic pruritus is not classically considered to be a neuropathic form of pruritus, it has been shown that $65 \%$ of patients with CKD exhibit peripheral nerve disorders, such as paresthesia or restless leg syndrome [20]. This has led to the hypothesis that uremic pruritus might actually be a product of damage to the peripheral 
nervous system, resulting in increased sensitivity to pruritogens [20]. Gabapentin has also been shown to significantly improve pruritus secondary to interleukin-2 (IL-2), theoretically by blocking pruritic stimulation at afferent nerves [7]. Regarding the use of pregabalin, case reports have described significant decreases in pruritus secondary to cetuximab and polycythemia vera, again expanding the utility of gabapentinoid therapy [7]. As evidence grows for the efficacy of gabapentinoids across multiple pruritic diseases, the case to use these medications to manage general pruritic conditions has become more compelling.

Research is also showing that other pruritic conditions classically not thought to be neuropathic in origin might indeed be able to attribute their pruritus to dysregulated nerves. For example, Takahashi et al. [21] showed that in normal skin, epidermal nerve endings are contained underneath keratinocyte tight junctions during epidermal turnover, but in human atopic dermatitis (AD) skin and in $\mathrm{AD}$ mouse models this nerve pruning is impaired, suggesting that these free nerve endings might contribute to itch in AD [21]. Studies have also found that patients with pruritus secondary to atopic predisposition and prurigo nodularis have an elevated density of SP-positive dermal nerve fibers [22-24] and that patients with pruritus-associated dermatologic conditions, such as $\mathrm{AD}$ and psoriasis, also have elevated cutaneous levels of SP [25-27]. Although there are no reports to date of gabapentin's utility for the management of chronic itch in $\mathrm{AD}$, the combined findings of increased SP and lack of nerve pruning in $\mathrm{AD}$ suggest that gabapentin might indeed be able to ameliorate pruritus in patients with $\mathrm{AD}$, whose itch is traditionally thought to be secondary to non-neuropathic causes.

\section{SAFETY PROFILE OF GABAPENTIN AND PREGABALIN IN OLDER ADULTS}

Although gabapentin and pregabalin are relatively safe drugs with benign side-effect profiles in the general population, both medications have several unique properties that must be taken into consideration when treating older adults. First and foremost, gabapentin and pregabalin are almost exclusively eliminated by the kidneys. The labels across all brands of gabapentin and pregabalin caution that since older adults are more likely to have decreased renal function, dose adjustments in this population should be made based on creatinine clearance [28-31]. A 2018 study of 140,899 adult hemodialysis patients (median age 61 years) who were receiving gabapentin or pregabalin found that when compared to no gabapentinoid use, gabapentin was associated with a 50\% higher hazard of altered mental status, 55\% higher hazard of fall, and 38\% higher hazard of fracture. Pregabalin was associated with a 51\% higher hazard of altered mental status and 68\% higher hazard of fall [32]. Thus, prior to gabapentin initiation, patients should be asked if they have compromised kidney function, and if they do, creatinine clearance should be evaluated and gabapentinoid dosage should be appropriately adjusted. Renal function should then be carefully monitored throughout their gabapentinoid course.

Some of the commonly reported side effects for both gabapentinoids were dizziness and somnolence [28-31], and these were the most common adverse reactions that led to discontinuation of gabapentin in clinical trials for postherpetic neuralgia and epilepsy [28, 30, 31]. While clinical trials with gabapentin did not find that older adults experienced these conditions at a higher incidence than younger study participants [28, 30, 31], trials evaluating pregabalin's efficacy in fibromyalgia did report that there were several neurological adverse reactions that were more frequent in older adults. These reactions included dizziness, blurred vision, problems with balance, tremor, confused state, abnormal coordination, and lethargy. Of note, this same increase was not seen in pregabalin trials for diabetic peripheral neuropathy or postherpetic neuralgia [29]. Therefore, while data might not consistently support that neurologic adverse reactions occur more frequently in older adults, it is nonetheless important to keep these in mind when prescribing gabapentinoids to this population. 
Patients should be asked if they already experience baseline dizziness or somnolence and warned that this could be exacerbated with gabapentinoid use.

Another condition that should be monitored when administering gabapentinoids is a possible increase in suicidal thoughts and behavior. This potential adverse event is part of the 2008 FDA class warning for antiepileptic drugs (AEDs). While studies have since found conflicting evidence on the link between AEDs and suicidality, some pooled analyses on AEDs have found an increased risk of suicidal ideation as early as 1 week after treatment initiation [33]; consequently, this warning is still listed in the drug information for both gabapentin and pregabalin [34]. Despite the contradictory data, this warning should not be dismissed in patients with $\mathrm{CP}$ since even at baseline, studies have suggested that pruritus can be as debilitating as chronic pain [35], potentially contributing to the strong association that has been found between $\mathrm{CP}$ and comorbid anxiety and depression with a high risk of suicidal ideation [36]. In fact, Hawro et al. [37] found that while suicidal ideation was prevalent among $11.8 \%$ of study participants with $\mathrm{AD}$, this percentage jumped to $18.5 \%$ of study participants with CP [37]. Additionally, since pruritus often worsens in the evenings, the perpetual need to itch can keep patients up at night, reducing both the quality and quantity of their sleep and thereby possibly exacerbating comorbid mood disorders. Among older adults, especially those who already suffer from inadequate support groups, chronic medical illness, or depression, the impact of $\mathrm{CP}$ on quality of life and mental wellbeing can be even further compounded, especially given that $\mathrm{CP}$ is refractory to treatment [3] in many patients. Therefore, in a patient population that already has increased rates of depression, anxiety, and suicidality due to their CP [36], physicians need to be mindful of potentially worsening these mood disorders by prescribing gabapentin or pregabalin. Patients should consequently be screened for mood disorders and warned of possible mood changes prior to gabapentinoid initiation.

While there has been no evidence to suggest differing efficacy of gabapentinoids between older and younger adults [28-31], one notable example of differences in safety was observed during clinical trials for gabapentinoid use in postherpetic neuralgia. Older age was associated with an increased incidence of peripheral edema (seen in trials for both Neurontin and Gralise $[28,30]$ ) and ataxia (seen in trials for Neurontin [28]); this association was not seen in trials for pregabalin [29]. Peripheral edema has been observed in several randomized control trials for gabapentin [38], and case reports suggest that this finding can be observed within days to 2 weeks of initiation [39-41]. Providers should counsel patients on this possible reaction in advance of gabapentin initiation so that patients are not alarmed if peripheral edema does develop.

Perhaps, however, the most important consideration when prescribing gabapentinoids to older adults is their interaction with opioids. This was included in the 2019 update to the Beers criteria [8] and issued as a safety alert by the FDA in December 2019 [42]. The Beers criteria state that use of opioids in concurrence with gabapentinoids should be avoided (unless transitioning from opioids to gabapentinoids) due to the increased risk of severe sedation-related adverse events, such as respiratory depression [8]. The FDA warning further elaborates that this risk applies not just to patients on opioids, but also to those who are on other central nervous system (CNS) depressants or who have a history of reduced lung function from conditions such as chronic obstructive pulmonary disease [42]. Patients should thus be thoroughly screened for reduced lung function and use of opioids or CNS depressants; if any of these serve as a contraindication to gabapentinoid use, then other treatments should be considered.

Lastly, prescribers should be aware of the gabapentinoids' potential for abuse. Pregabalin is a Schedule $\mathrm{V}$ controlled substance, and although gabapentin is not listed as a controlled substance, there is a growing body of literature on its potential for misuse, especially among patients with a history of substance abuse. In a study of 503 opioid abusers not in treatment, $15 \%$ said that they used gabapentin within the past 6 months to get high [43]. Abuse reported 
in case reports was noted with doses ranging from 1500 to $1200 \mathrm{mg}$, with many patients reporting that they self-titrated the medication [44]. Gabapentinoid screening should therefore involve a thorough history of substance use to evaluate for propensity for drug abuse.

\section{CONSIDERATIONS/ RECOMMENDATIONS FOR GABAPENTINOID USE IN OLDER ADULTS}

The authors recommend that when considering gabapentinoids for pruritic patients, screening questions should be asked to verify safety in administration. If screened appropriately without contraindication (Table 1), an aging patient should be prescribed a low initial dose with close follow-up to ensure tolerance. While both gabapentin and pregabalin can be used in the treatment of $\mathrm{CP}$, gabapentin is more often prescribed since pregabalin is a Schedule $\mathrm{V}$ controlled substance and, due to its sedative side effects or lack of efficacy, healthcare coverage may be difficult to obtain without evidence of failure of gabapentin. Therefore, the recommendations we detail below for gabapentinoid use in CP are specifically for gabapentin. The provided dose recommendations should be tailored to the individual patient's renal function. The dosage suggestions outlined below are based on expert recommendations, which admittedly are variable and idiosyncratic per patient response. The pharmacokinetics for both drugs are detailed in Table 2.

When gabapentin is dosed for the management of chronic pain, the goal is to maintain a steady state in the body to provide adequate pain relief throughout the day; for itch, however, the goal of treatment is to decrease nocturnal pruritus to maximize sleep. The first dose of gabapentin should therefore be given at night. Gabapentin is metabolized slowly, with an elimination half-life of $5-7 \mathrm{~h}$ that is not affected by dose [28]. Due to this slow metabolism, administering an adequate dose of gabapentin before sleep should help provide relief from pruritus throughout the night. The authors recommend an initial nighttime dose of $100 \mathrm{mg}$ that can be titrated up to $300 \mathrm{mg}$ within weeks. If the patient still experiences intractable itch while on $300 \mathrm{mg}$, a second

Table 1 Screening questions for older adults on gabapentinoids

\section{Prior to gabapentinoid initiation}

Do you have a history of kidney disease? (check creatinine clearance if concern for poor renal function)

Do you have a history of falls, lightheadedness, or vertigo?

Do you have a history of mood disorders including anxiety, depression, and suicidal ideation?

Do you have a history of chronic obstructive pulmonary disease (COPD) or other lung disorders?

Are you currently taking any opioid medications?

Do you have a history of recreational drug usage?

\section{At 1-2 weeks after initiation or dose change}

After starting your medication have you had any changes in mood, including new or increased depression, anxiety, or suicidal ideation?

After starting your medication have you experienced any dizziness, fatigue, or blurred vision?

Have you developed any swelling in your arms or legs?

Have you had difficulty balancing or felt uncoordinated? 
Table 2 Gabapentin and pregabalin pharmacokinetics and drug interactions

\begin{tabular}{llc}
\hline $\begin{array}{l}\text { Pharmacokinetics and drug } \\
\text { interactions }\end{array}$ & Gabapentin & Pregabalin \\
\hline Half-life & $5-7 \mathrm{~h}$ & $6 \mathrm{~h}$ \\
Drug Interactions & Morphine increases concentrations & No known drug \\
& $\begin{array}{l}\text { Oral anatacids containing magnesium and aluminum hydroxide } \\
\text { reduce bioavailability by 20\% }\end{array}$ & interactions \\
Starting dosing & $100 \mathrm{mg}$ & $75 \mathrm{mg}$ \\
Timing for onset of action & Days & Weeks \\
\hline
\end{tabular}

Information in this table is taken from gabapentin and pregabalin US Food and Drug Adminisraton labels [28-31]

evening dose should be added. For example, the patient could take their first evening dose of $300 \mathrm{mg}$ at 5:00 p.m. and then their second dose of $300 \mathrm{mg}$ at 8:00 p.m. If the patient still has severe refractory nocturnal pruritus, then the 8:00 p.m. dose should be increased to $600 \mathrm{mg}$ while keeping the 5:00 p.m. dose at $300 \mathrm{mg}$ (Table 3). For patients whose pruritus is severe during the day, they can receive standard gabapentin dosing starting at $300 \mathrm{mg}$ three times daily with titration as needed to a maximum of $3600 \mathrm{mg} /$ day [45]. Once symptoms are adequately controlled, patients can remain on a maintenance dose of gabapentin. If the decision is made to stop gabapentin, it is recommended to taper the dose off gradually over 1 week or longer $[28,30]$. If gabapentin is abruptly discontinued, case reports suggest that patients can experience withdrawal. In most cases, withdrawal symptoms developed between 24 to $48 \mathrm{~h}$ of discontinuation, but symptoms have been reported as late as 7 days after cessation. Reported withdrawal symptoms include agitation, confusion, disorientation, diaphoresis, and gastrointestinal symptoms. These symptoms resolved upon re-initiation of gabapentin [46].

Regarding the use of pregabalin for the treatment of $\mathrm{CP}$, an open label trial of 22 patients (aged $56 \pm 13.65$ years) observed a significant decrease of itch intensity 4 weeks after initiation of treatment with a fixed dose of $150 \mathrm{mg} /$ day; no difference in itch intensity was observed between 4 and 8 weeks [47]. These

Table 3 Gabapentin dosing in older adults

Gabapentin dosing for older adults with nocturnal chronic pruritus

Time Dosage

Initial dosing

8:00 p.m.

$100 \rightarrow 300 \mathrm{mg}$

Refractory nocturnal pruritus

5:00 p.m.

$300 \mathrm{mg}$

8:00 p.m.

$300 \mathrm{mg}$

Severe nocturnal pruritus

5:00 p.m. $\quad 300 \mathrm{mg}$

$\begin{array}{lll}8: 00 \text { p.m. } & 600 \mathrm{mg}\end{array}$ 
results led the authors to conclude that pregabalin's maximum antipruritic efficacy was at 4 weeks and that it could be used as maintenance therapy thereafter [47].

After starting gabapentinoids in an older patient, it is important to screen for adverse effects, as detailed in Table 1. Screening questions should be asked before initiation and at 1-2 weeks after initiation or dose change since suicidal symptoms and peripheral edema have been reported within days of starting gabapentin [33, 39-41]. Directed questions should be asked about dizziness, problems with balance, blurred vision, or fatigue. Patients who are specifically on Neurontin or Gralise should also be evaluated for peripheral edema and ataxia. Most importantly, any changes in mood should be assessed by asking about new or increased depression or suicidal ideation. If patients are on $>600 \mathrm{mg} /$ day physicians should also be aware of a potential increased risk of altered mental status. In a study evaluating 30-day risk of hospitalization with altered mental status (defined by urgent head computed tomography [CT] examination without indication of stroke) and mortality in older adults after initiating gabapentin, it was found that a dose $>600 \mathrm{mg} /$ day was associated with a higher risk of hospitalization with head CT scan compared to $\leq 600 \mathrm{mg} /$ day, but not a higher risk of mortality [48].

\section{CONSIDERATIONS FOR THE FUTURE OF TREATING ITCH IN OLDER ADULTS}

The current model of the itch-scratch cycle paints a multifactorial picture of pruritus: scratching causes an epithelial stress response that perpetuates inflammation via cytokine release, and keratinocytes activate itch-sensory neurons that release substance $P$ and CGRP which in turn cause neurogenic inflammation [49]. The theory that gabapentinoids are efficacious in CP by modulating neuropeptides that activate immune cells to cause inflammation, highlights a complex interplay between the body's nervous and immune systems. Given this potential intersection between neurogenic and immune mediated itch, it is therefore possible that gabapentinoids may alleviate itch even in patients whose CP is not exclusively neuropathic in origin.

Data to date are conflicting [50,51], but they may suggest that gabapentinoids could serve as a wide-reaching treatment for pruritus with a favorable efficacy and side-effect profile compared to ubiquitous medications like antihistamines. Although antihistamines have limited efficacy for managing CP and pose considerable risk to older adults, the lack of consensus on a treatment algorithm for $\mathrm{CP}$ in this population often leads to undertreatment with the use of topicals or antihistamines. To better understand this practice gap in treating older patients with $\mathrm{CP}$, the field needs to continue to investigate effective treatments, management strategies, and underlying mechanisms. However, studies and clinical trials often neglect to adequately include older adults [52], leaving providers in the dark as to the safety and efficacy of treatments in this population. In light of this gap, not only must treatments for $\mathrm{CP}$ be investigated, but these investigations must deliberately focus on the efficacy and safety of therapies among older adults. Until then, observational research will serve as the first step to build a broader framework for teaching and discovering best practices.

\section{CONCLUSION}

Gabapentin and pregabalin stand as efficacious and safe options for the treatment of multifactorial pruritus in older adults. The aim of this review is to provide context to the nuances of prescribing these medications in this population and advocates for the inclusion of these medications in the limited therapeutic ladder for $\mathrm{CP}$ in older adults. While the field of dermatology continues to evaluate best practices in this population, it is essential for providers to be educated and confident in using these potential options. 


\section{ACKNOWLEDGEMENTS}

Funding. No funding or sponsorship was received for this study or publication of this article.

Authorship. All named authors meet the International Committee of Medical Journal Editors (ICJME) criteria for authorship for this article, take responsibility for the integrity of the work as a whole, and have given their approval for this version to be published.

Disclosures. Shreya Sreekantaswamy, Nicholas Mollanazar, and Daniel Butler declare that they have no conflicts of interest.

Compliance with Ethics Guidelines. This article is based on previously conducted studies and does not contain any studies with human participants or animals performed by any of the authors.

Data Availability. Data sharing is not applicable to this article as no datasets were generated or analyzed during the current study.

Open Access. This article is licensed under a Creative Commons Attribution-NonCommercial 4.0 International License, which permits any non-commercial use, sharing, adaptation, distribution and reproduction in any medium or format, as long as you give appropriate credit to the original author(s) and the source, provide a link to the Creative Commons licence, and indicate if changes were made. The images or other third party material in this article are included in the article's Creative Commons licence, unless indicated otherwise in a credit line to the material. If material is not included in the article's Creative Commons licence and your intended use is not permitted by statutory regulation or exceeds the permitted use, you will need to obtain permission directly from the copyright holder. To view a copy of this licence, visit http://creativecommons.org/licenses/by$\mathrm{nc} / 4.0 /$.

\section{REFERENCES}

1. Weisshaar E, Dalgard F. Epidemiology of itch: adding to the burden of skin morbidity. Acta Derm Venereol. 2009;89:339-50.

2. Ständer S, Weisshaar E, Mettang T, et al. Clinical classification of itch: a position paper of the International Forum for the Study of Itch. Acta Derm Venereol. 2007;87(4):291-4.

3. Weisshaar E, Szepietowski J, Dalgard FJ, et al. European S2k guideline on chronic pruritus. Acta Derm Venereol. 2019;99(5):469-506.

4. Berger TG, Shive M, Harper GM. Pruritus in the older patient: a clinical review. JAMA. 2013;310(22):2443-50.

5. Valdes-Rodriguez R, Stull C, Yosipovitch G. Chronic pruritus in the elderly: pathophysiology, diagnosis and management. Drugs Aging. 2015;32(3):201-15.

6. Stumpf A, Ständer S. Neuropathic itch: diagnosis and management. Dermatol Ther. 2013;26(2): 104-9.

7. Matsuda KM, Sharma D, Schonfeld AR, Kwatra SG. Gabapentin and pregabalin for the treatment of chronic pruritus. J Am Acad Dermatol. 2016;75(3): 619-25.

8. 2019 American Geriatrics Society Beers Criteria ${ }^{\circledR}$ Update Expert Panel. American Geriatrics Society 2019 Updated AGS Beers Criteria ${ }^{\circledR}$ for Potentially Inappropriate Medication Use in Older Adults. J Am Geriatr Soc. 2019;67(4):674-94.

9. Yosipovitch G, Rosen JD, Hashimoto T. Itch: From mechanism to (novel) therapeutic approaches. J Allergy Clin Immunol. 2018;142(5):1375-90.

10. Kouwenhoven TA, van de Kerkhof P, Kamsteeg M, Kamsteeg M. Use of systemic treatment in patients with chronic pruritus: a survey of dermatologists in the Netherlands. Acta Derm Venereol. 2019;99(3): 304-8.

11. Fukada C, Kohler JC, Boon H, Austin Z, Krahn M. Prescribing gabapentin off label: perspectives from psychiatry, pain and neurology specialists. Can Pharm J (Ott). 2012;145(6):280-4.e1.

12. Taylor CP, Angelotti T, Fauman E. Pharmacology and mechanism of action of pregabalin: the calcium channel $\alpha 2-\delta$ (alpha2-delta) subunit as a target for antiepileptic drug discovery. Epilepsy Res. 2007;73(2):137-50.

13. Luo ZD, Chaplan SR, Higuera ES, et al. Upregulation of dorsal root ganglion (alpha)2(delta) calcium 
channel subunit and its correlation with allodynia in spinal nerve-injured rats. J Neurosci. 2001;21(6): 1868-75.

14. Lee S. Pharmacological inhibition of voltage-gated $\mathrm{Ca}(2+)$ channels for chronic pain relief. Curr Neuropharmacol. 2013;11(6):606-20.

15. Fehrenbacher JC, Taylor CP, Vasko MR. Pregabalin and gabapentin reduce release of substance $P$ and CGRP from rat spinal tissues only after inflammation or activation of protein kinase C. Pain. 2003;105(1):133-41.

16. Takasusuki T, Yaksh TL. The effects of intrathecal and systemic gabapentin on spinal substance $P$ release. Anesth Analg. 2011;112(4):971-6.

17. Bueller HA, Bernhard JD, Dubroff LM. Gabapentin treatment for brachioradial pruritus. J Eur Acad Dermatol Venereol. 1999;13(3):227-8.

18. Ehrchen J, Ständer S. Pregabalin in the treatment of chronic pruritus. J Am Acad Dermatol. 2008;58(2 Suppl 1):S36-7.

19. Yesudian PD, Wilson NJE. Efficacy of gabapentin in the management of pruritus of unknown origin. Arch Dermatol. 2005;141(12):1507-9.

20. Eusebio-Alpapara KMV, Castillo RL, Dofitas BL. Gabapentin for uremic pruritus: a systematic review of randomized controlled trials. Int J Dermatol. 2020;59(4):412-22.

21. Takahashi S, Ishida A, Kubo A, et al. Homeostatic pruning and activity of epidermal nerves are dysregulated in barrier-impaired skin during chronic itch development. Sci Rep. 2019;9(1):8625.

22. Haas S, Capellino S, Phan NQ, et al. Low density of sympathetic nerve fibers relative to substance P-positive nerve fibers in lesional skin of chronic pruritus and prurigo nodularis. J Dermatol Sci. 2010;58(3):193-7.

23. Järvikallio A, Harvima IT, Naukkarinen A. Mast cells, nerves and neuropeptides in atopic dermatitis and nummular eczema. Arch Dermatol Res. 2003;295(1):2-7.

24. Molina FA, Burrows NP, Jones RR, Terenghi G, Polak JM. Increased sensory neuropeptides in nodular prurigo: a quantitative immunohistochemical analysis. Br J Dermatol. 1992;127(4): 344-51.

25. Wallengren J, Ekman R, Ekman R, Sundler F. Occurrence and distribution of neuropeptides in the human skin. An immunocytochemical and immunochemical study on normal skin and blister fluid from inflamed skin. Acta Derm Venereol. 1987;67(3):185-92.

26. Pincelli C, Fantini F, Massimi P, Girolomoni G, Seidenari S, Giannetti A. Neuropeptides in skin from patients with atopic dermatitis: an immunohistochemical study. Br J Dermatol. 1990;122(6): $745-50$.

27. Chan J, Smoller B, Raychauduri SP, Jiang WY, Farber EM. Intraepidermal nerve fiber expression of calcitonin gene-related peptide, vasoactive intestinal peptide and substance $P$ in psoriasis. Arch Dermatol Res. 1997;289(11):611-6.

28. Pzifer Inc. Neurontin (gabapentin) [package insert]. Highlights of prescribing information. U.S. Food and Drug Administration website. 2017. https:// www.accessdata.fda.gov/drugsatfda_docs/label/ 2017/020235s064_020882s047_021129s046lbl.pdf. Accessed 21 October 2020.

29. Pzifer Inc. Lyrica (pregabalin) [package insert]. Highlights of prescribing information. U.S. Food and Drug Administration website. 2011. https:// www.accessdata.fda.gov/drugsatfda_docs/label/ 2011/021446s026,022488s005lbl.pdf.Accessed 21 October 2020.

30. Almatica Pharma. Gralise (gabapentin) [package insert]. Highlights of prescribing information. U.S. Food and Drug Administration website. 2015. https://www.accessdata.fda.gov/drugsatfda_docs/ label/2020/022544s026lbl.pdf. Accessed 21 October 2020.

31. Patheon N.V. (Thermo Fisher Scientific). Horizant (gabapentin) [package insert]. Highlights of prescribing information. U.S. Food and Drug Administration website. 2012. https://www.accessdata.fda. gov/drugsatfda_docs/label/2012/

022399s006,s007lbl.pdf. Accessed 21 October 2020.

32. Ishida JH, McCulloch CE, Steinman MA, Grimes BA, Johansen KL. Gabapentin and pregabalin use and association with adverse outcomes among hemodialysis patients. J Am Soc Nephrol. 2018;29(7):1970-8.

33. Lexicomp. Gabapentin: Drug Information UpToDate. https://www.uptodate.com/contents/gabape ntin-drug-information?search=Gabapentin\&source =panel_search_result\&selectedTitle=1 149\&usage _type=panel\&kp_tab=drug_general\&display_rank= 1\#F174988. Accessed 24 October 2020.

34. Gibbons RD, Hur K, Brown CH, Mann JJ. Gabapentin and suicide attempts. Pharmacoepidemiol Drug Saf. 2010;19(12):1241-7.

35. Kini SP, DeLong LK, Veledar E, McKenzie-Brown AM, Schaufele M, Chen SC. The impact of pruritus 
on quality of life: the skin equivalent of pain. Arch Dermatol. 2011;147(10):1153-6.

36. Stumpf A, Schneider G, Ständer S. Psychosomatic and psychiatric disorders and psychologic factors in pruritus. Clin Dermatol. 2018;36(6):704-8.

37. Hawro T, Przybyłowicz K, Spindler M, et al. The characteristics and impact of pruritus in adult dermatologic patients: A prospective, cross-sectional study. J Am Acad Dermatol. 2021;84(3):691-700.

38. Zhang M, Gao C-X, Ma K-T, et al. A meta-analysis of therapeutic efficacy and safety of gabapentin in the treatment of postherpetic neuralgia from randomized controlled trials. Biomed Res Int. 2018;2018: 7474207.

39. Bidaki R, Sadeghi Z, Shafizadegan S, et al. Gabapentin induces edema, hyperesthesia and scaling in a depressed patient; a diagnostic challenge. Adv Biomed Res. 2016;5:1.

40. Finegan A, Mabrouk U, Nelson LA. Gabapentin-induced bilateral lower extremity edema in a patient with pervasive developmental disorder and schizoaffective disorder. Ment Health Clin. 2020;10(4):250-3.

41. Kahlon A, Gnanabakthan N, Dhillon A, Subedi D. A rare case of bilateral lower extremity edema due to low dose gabapentin therapy in a young male patient. J Basic Clin Pharm. 2015;6(4):117-8.

42. U.S. Food and Drug Administration. FDA warns about serious breathing problems with seizure and nerve pain medicines gabapentin (Neurontin, Gralise, Horizant) and pregabalin (Lyrica, Lyrica CR). 2019. https://www.fda.gov/drugs/drug-safety-andavailability/fda-warns-about-serious-breathingproblems-seizure-and-nerve-pain-medicinesgabapentin-neurontin. Accessed 24 October 2020.

43. Smith RV, Lofwall M, Havens JR, Havens JR. Abuse and diversion of gabapentin among nonmedical prescription opioid users in Appalachian Kentucky. Am J Psychiatry. 2015;172(5):487-8.

44. Evoy KE, Morrison MD, Saklad SR. Abuse and misuse of pregabalin and gabapentin. Drugs. 2017;77(4):403-26.

45. Steinhoff M, Cevikbas F, Ikoma A, Berger TG. Pruritus: management algorithms and experimental therapies. Semin Cutan Med Surg. 2011;30(2): 127-37.

46. Mersfelder TL, Nichols WH. Gabapentin: abuse, dependence, and withdrawal. Ann Pharmacother. 2016;50(3):229-33.

47. Park J-M, Jwa S-W, Song M, et al. Efficacy and safety of pregabalin for the treatment of chronic pruritus in Korea. J Dermatol. 2012;39(9):790-1.

48. Fleet JL, Dixon SN, Kuwornu PJ, et al. Gabapentin dose and the 30-day risk of altered mental status in older adults: A retrospective population-based study. PLoS ONE. 2018;13(3):e0193134-e.

49. Mack MR, Kim BS. The itch-scratch cycle: a neuroimmune perspective. Trends Immunol. 2018;39(12):980-91.

50. Gobo-Oliveira M, Pigari VG, Ogata MS, Miot HA, Ponce D, Abbade LP. Gabapentin versus dexchlorpheniramine as treatment for uremic pruritus: a randomised controlled trial. Eur J Dermatol. 2018;28(4):488-95.

51. Ancona-Castro C, Navarrete-Solís J, et al. Efficacy of gabapentin compared to hydroxyzine in the treatment of chronic pruritus. Dermatol Rev Mex. 2018;62(6):486-96.

52. Butler DC. Dermatology's management of flagship diseases in older adults: lifting the blindfold. JAMA Dermatol. 2020;156(11):1175-6 